\title{
Recurrent flare/CME events from an emerging flux region
}

\author{
Nariaki V. Nitta \\ Lockheed Martin Solar and Astrophysics Laboratory, Palo Alto, California \\ Hugh S. Hudson \\ Solar Physics Research Corporation, ISAS, Sagamihara, Japan
}

\begin{abstract}
We report on six recurrent 'halo' coronal mass ejections (CMEs) that occurred (in November 2000) during a 60-hour period in clear association with major flares in an active region on the solar disk. The region was undergoing dynamic restructuring due to flux emergence. The flares were not long-decay events (LDEs) in terms of soft X-ray light curves and morphologies, although, in the impulsive phase, they produced ejections in soft X-rays that are characteristic of CMEs. We do not detect global changes in EUV and X-ray full-disk images prior to these flares. We suggest that emerging magnetic flux in the core of an active region may be responsible for the occurrence of such repeated flare/CME events.
\end{abstract}

\section{Introduction}

Some coronal mass ejections (CMEs) are associated with filament eruptions in quiescent regions. We empirically know, however, that CMEs associated with flares in active regions are also common (e.g., Munro et al. [1979]). Based on the acceleration profiles and morphologies, there seem to be two types of CMEs [Sheeley et al., 1999], and it is likely that those which start fast and then decelerate correspond to flares more directly (cf., MacQueen and Fisher [1983]). Fast CMEs may produce interplanetary shocks that are considered to be responsible for major solar energetic particle (SEP) events observed near Earth [Reames, 1999].

CMEs tend to accompany gradual X-ray brightenings [Sheeley et al., 1975], which are often classified as flares when they exceed a certain X-ray flux threshold. Such events tend to have long decay times, hence they are called long decay events (LDEs) [Kahler, 1977]. According to Kahler et al. [1989], however, about $20 \%$ of the major impulsive flares are also associated with CMEs, which typically have narrow angular widths.

In this Letter, we report recurrent CMEs observed during a short period. All of them were clearly associated with major flares that were not LDEs. Unlike the tendency of CMEs associated with impulsive flares as found by Kahler et al. [1989], these CMEs were extended events. The associated flares all came from the same active region and occurred around the preceding spot. This area of the active region showed continual restructuring due to flux emergence. These events remind us that flare development often cannot

Copyright 2001 by the American Geophysical Union.

Paper number?.

0094-8276/01/?\$05.00 be distinguished from CME formation, even with the better resolution provided by modern data. We suggest here that the emergence of flux in the core of an active region may lead directly to the occurrence of a class of CMEs.

\section{Observations}

In a 60-hour interval starting 04:50 UT on 2000 November 24 , a total of five X-class flares occurred in NOAA active region (AR) 9236 (at heliographic latitude N18), which passed central meridian just before the first flare. Shortly after each of these flares and an additional M-class flare (labeled as $a^{-}$ $f$ ), the Large Angle Spectroscopic Coronagraph (LASCO) [Brueckner et al., 1995] on the Solar and Heliospheric Observatory (SOHO) recorded extended events, known as 'halo' CMEs [Howard et al., 1982]. Figure 1 shows the CMEs as observed with the LASCO C2 telescope, which observes the outer corona up to $\sim 6 R_{\odot}$. Even though the bright part of the CME tends to be confined on the west limb, all of them are included in the halo CME list of the LASCO team. Indeed, with a proper scaling, we can see extended emission at a low level. For each flare, we show in Figure 2 soft and hard $\mathrm{X}$-ray light curves from the GOES X-ray monitor (1-8 $\AA$ ) and the $\mathrm{L}(14-23 \mathrm{keV})$ and M2 (33-53 keV) channels of the Yohkoh Hard X-ray Telescope (HXT) [Kosugi et al., 1991].

In table 1 the start and end times indicate the times at which the GOES 1-8 $\AA$ flux crossed $5 \%$ of the peak flux. None of the flares are LDEs as defined by Kahler [1977], except for the marginal case of event $d$. The table also gives the longitude, the GOES class and the time of the first C2 image that shows the CME. Because of the $\sim 30$ minute cadence of LASCO, the plane-of-the-sky height of the first CME image varies from event to event. The presence of associated metric type II bursts is indicated in the next column, as taken from the NOAA reports. These have some ambiguity because the identification of a weak metric burst may not be made completely objectively. For example, a closer look at the data from event $d$ indicates a type II burst (A. Klassen and R. Gorgutsa, private communications, 2001), although the NOAA report makes no remark on a metric burst. The CME speeds in the plane of the sky are taken from the LASCO Web site and should be regarded as preliminary estimates. Interplanetary particle data from GOES 8 show small increases in the $\geq 10 \mathrm{MeV}$ proton flux for the first two events ( 7 and 70 particles $/ \mathrm{cm}^{2} / \mathrm{s} / \mathrm{sr}$ ), and these SEPs appear to have been accelerated by CME-driven interplanetary shocks (D. Reames, private communication, 2001).

We note that there was an M8 flare in another region AR 9240 (at E49) between flares $c$ and $d$. For comparison, 


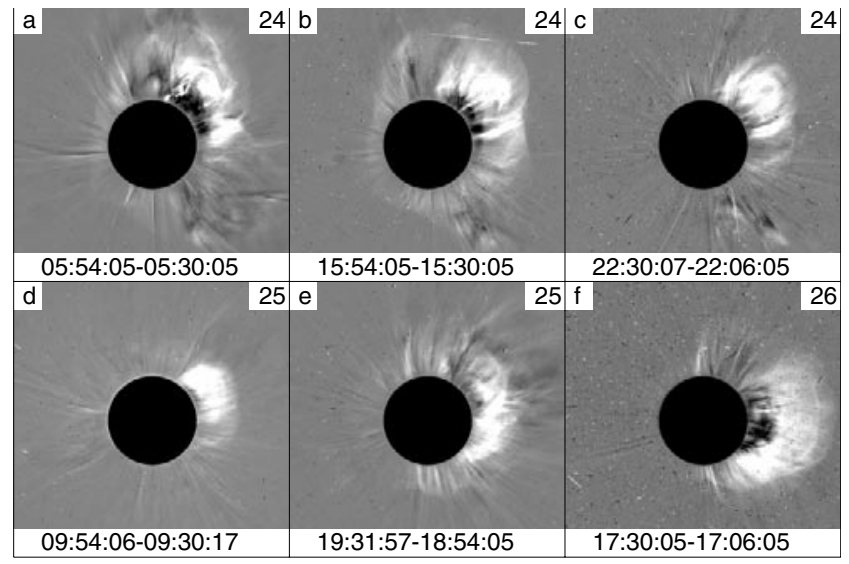

Figure 1. LASCO C2 difference images showing CMEs associated with six flares that occurred in AR 9236. The occulting disk, which has an effective radius of $2 R_{\odot}$, is shown in black.

we label this flare as $g$. Compared with flares $a-f$, this flare had more gradual time profiles, and it was in the LDE category. It was accompanied by a metric type II as well as type IV burst, and its associated CME was very fast. We attribute to this CME the interplanetary proton flux that started increasing gradually at mid-day November 25, peaking late on the 26th. Indeed, the LASCO images 1-2 hours before event $f$ were already noisy with particle tracks. Because of the elevated proton flux, it might not have been possible to see additional protons from event $f$.

In Figure 3, we show for each flare an image taken by the Yohkoh Soft X-ray Telescope (SXT) [Tsuneta et al., 1991] early in the event, overplotted with an HXT image, in comparison with a a magnetogram from the Michelson-Doppler Imager (MDI) [Scherrer et al., 1995] on SOHO. The first flare was almost completely missed by Yohkoh due to spacecraft night, so we plot the intensity of a $1600 \AA$ image from the Transition Region and Coronal Expolorer (TRACE) [Handy et al., 1999], which can be used as proxy for hard X-ray foot-point emission. All of the flares occurred around the preceding spot (positive polarity) of AR 9236. The first four flares involved the western part of the spot, and energetically peaked at flare $b$. It was clearly a white-light flare, as seen in TRACE white-light images, even though they have contributions from photons at shorter wavelengths. It also showed $\gamma$-ray line emission, based on the Yohkoh GammaRay Spectrometer data. Flares $c$ and $d$ were less energetic. The last two flares occurred at the southern part of the spot. The tilt of the hard X-ray foot-points also changed to the northwest-southeast orientation. Correspondingly, the brightest parts of the CMEs moved southward (Figure 1). The images in Figure 3 support in detail the tendency of these flares towards homology, i.e., the repetition of similar structures. This figure also shows the emergence of new magnetic flux, including the negative polarity (black) appearing at the south in frames $\mathrm{d}-\mathrm{f}$.

We note that flares $a-f$ were compact, not reminiscent of an arcade with a cusp, which often signifies an LDE. In contrast, flare $g$ (an LDE in the eastern hemisphere) showed an extended arcade. Therefore, flares $a-f$ were morphologically not LDEs. Based solely on the soft X-ray time profiles, which all show that the rise time is much shorter than the decay time, one might argue that there is no fun- damental difference between flares $a-f$ and flare $g$, but the morphologies suggest that this is not the case.

Despite the lack of the LDE characteristics, flares $a-f$ all involved ejections seen in soft X-rays in the large fieldof-view images of SXT. Such flare ejecta are known to be correlated with CMEs [Nitta and Akiyama, 1999] and may contribute to the CME mass. In addition, the highcadence TRACE $1600 \AA$ data captured ejections in flare $b$, as did ground-based $\mathrm{H} \alpha$ observations at least in flares $a$ and $c$ (R. Kitai and H. Wang, private communications, 2001). For each flare, dimming was observed in images from the Extreme-Ultraviolet Imaging Telescope (EIT) [Delaboudinière et al., 1995] on SOHO. The dimming areas were always southeast (stronger) and west to northwest (weaker) from the preceding spot.

We have put MDI magnetograms with a 96 minute cadence into movie form, and noted the very dynamic changes around the preceding spot that took place before and during the period of these flares. Many small polarity pairs (bipoles) can be seen appearing in the MDI data. Eventually these formed simpler spots to the south and northeast of the preceding spot (see Figure 3). Because of encounters of opposite-polarity elements, there must have been many flux cancellation events, and, as shown in Figure 4, the changes certainly involved the emergence of new flux during the interval in which these flares occurred. We do not see obvious sudden changes in the magnetic maps due to the flares.

Finally, we examined all the SXT and EIT full disk images during November 24-26, again putting each set into movie form. This was primarily to see if there were preevent changes on a global scale that may have caused both the flares and CMEs (cf., Low [1996]). We also tried to see if there was a filament eruption elsewhere, which could confuse any attempt to correlate CMEs with flares. We find no remarkable changes in the SXT and EIT data during the period of $\sim 1.5$ hours before the onset of each flare, within the limit of their cadence and data coverage, and that the CME development in each case proceeded with its associated flare.
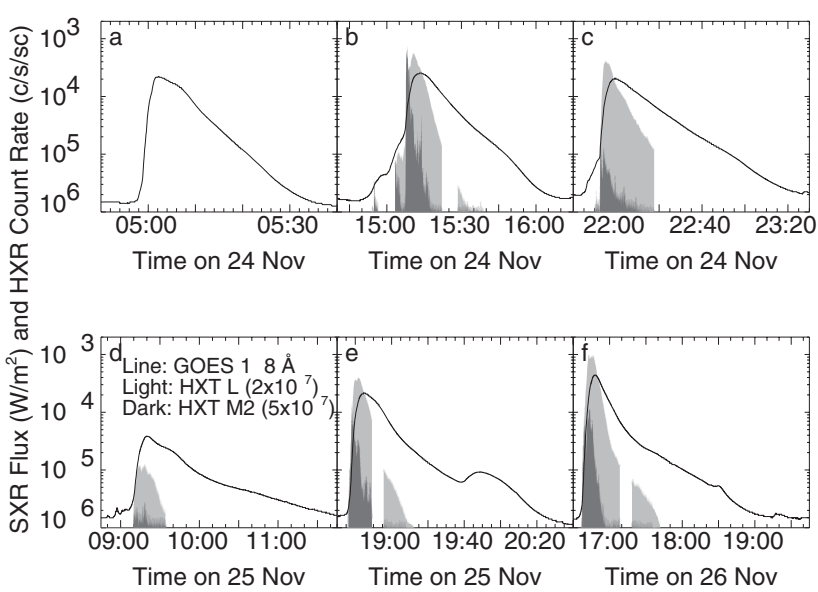

Figure 2. Light curves of the six flares whose associated CMEs are shown in Figure 1. The solid lines show the GOES 1-8 flux. The filled areas show HXT count rates (light: L channel, dark: M2 channel). 
Table 1. Information on flares

\begin{tabular}{rrrrrrrrrrr}
\hline ID & Day & Start & Peak & End & Longitude & GOES & C2 & Type II & $V_{C M E^{\text {a }}}$ Protons \\
\hline a & 24 & $04: 59$ & $05: 02$ & $05: 20$ & W05 & X2.0 & $05: 30$ & yes & 711 & yes \\
b & 24 & $15: 05$ & $15: 13$ & $15: 44$ & W11 & X2.3 & $15: 30$ & yes & 890 & yes \\
c & 24 & $21: 53$ & $22: 00$ & $22: 49$ & W15 & X1.8 & $22: 06$ & $?^{\mathrm{b}}$ & 935 & no \\
d & 25 & $09: 09$ & $09: 20$ & $10: 54$ & W21 & M3.5 & $09: 30$ & $?^{\text {c }}$ & 610 & no \\
e & 25 & $18: 38$ & $18: 45$ & $19: 22$ & W24 & X1.9 & $19: 31$ & yes & 596 & no \\
f & 26 & $16: 40$ & $16: 48$ & $17: 31$ & W36 & X4.0 & $17: 06$ & yes & 869 & $?^{\mathrm{d}}$ \\
g & 25 & $01: 02$ & $01: 31$ & $03: 55$ & E49 & M8.2 & $01: 31$ & yes & 2202 & yes \\
\hline
\end{tabular}

${ }^{\mathrm{a}} \mathrm{In} \mathrm{km} / \mathrm{s}$.

${ }^{\mathrm{b}}$ Continuum burst, according to the NOAA report.

${ }^{c}$ Possible type II burst, according to A. Klassen (Potsdam) and R. Gorgutsa (IZMIRAN).

${ }^{\mathrm{d}}$ High background level.

\section{Discussion and Conclusions}

We have reported the occurrence of impulsive and homologous flares closely associated with CME formation. This reminds us of the existence of a class of CMEs, which cannot be understood properly without studying the associated flare. However, it appears that flares are not considered seriously in the existing CME models. We point out several factors that may have made CME modelers generalize that flares are unimportant. First, a major CME can occur without an X-ray brightening strong enough to be classified as a flare. The low atmospheric signatures of some halo CMEs can be tiny filament eruptions, which are not easily distinguished from those that frequently occur all over the Sun. Second, even though LDEs are usually associated with CMEs, the association of impulsive flares with CMEs is not high [Kahler et al., 1989]. Third, CMEs tend to involve much larger areas than an average active region which
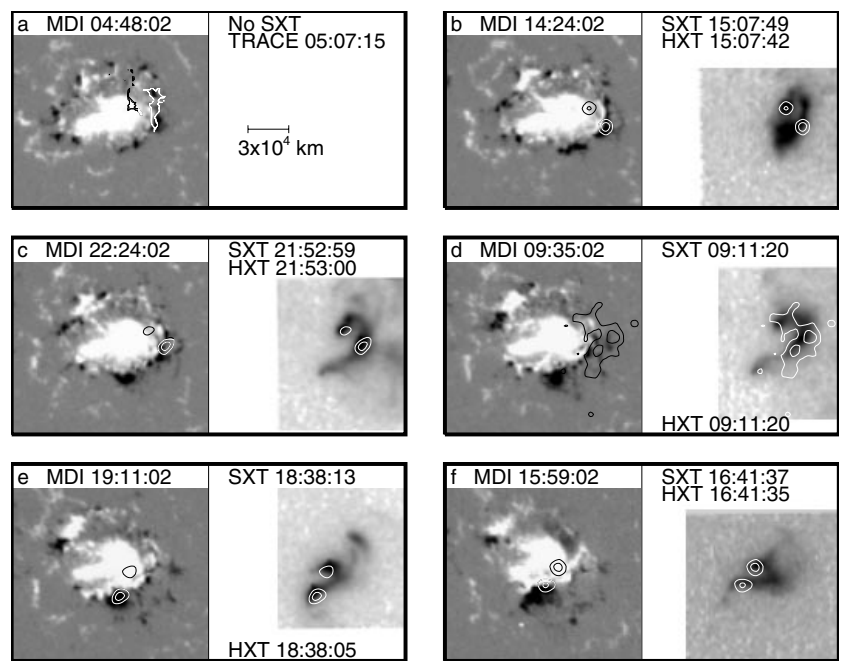

Figure 3. Line-of-sight magnetograms (from MDI) and soft $\mathrm{X}$-ray images (from SXT) for the six flares in Figure 2. These images cover only the preceding spot of AR 9236. The white (black) areas in the magnetograms correspond to the positive (negative) polarity. The SXT images are shown in reverse color. The contours show hard X-ray images from the HXT M2 channel (M1 channel for flare $d$ ), and the contour levels are $15 \%$ and $50 \%$ of the peak of each image. For the first event, whose main phase was not observed by Yohkoh, the TRACE $1600 \AA$ data are shown as proxy to hard X-ray emission. As usual, solar north is up and west is to the right. is in turn larger than a flare. Moreover, traditional backprojection of the CME height has put the CME onset time earlier than the flare onset. Lastly, interplanetary and geomagnetic disturbances are attributed more directly to CMEs than to flares (e.g., Gosling [1993]).

We argue that none of these factors preclude the possible importance of flare-related processes, such as blast waves and shocks, for the initiation of the CMEs in our six events, or at least the possible link between the initiation of the two phenomena. The occurrence of CMEs without flare associations and impulsive flares without CMEs simply establishes a type of CME for which X-ray brightening (due to heating) is only of secondary importance. In this type, a flare (LDE) would result, if the magnetic field is strong, as in an active region [Svestka, 2001]. Impulsive flares look morphologically different from LDEs, and it is possible that different processes are operative in producing compact flares and LDEs. Whether an impulsive flare is associated with a CME may depend on its magnetic configuration, as we speculate in the following. If it occurs under strong closed fields, the disturbances may not have long-range effects, and so the flare would appear to be confined. In the case of our flares $a-f$, they occurred on the western or southern side of the preceding spot, where, unlike on the eastern side, the field lines did not close to the following plage region but connected to remote areas, as indicated in large field-of-view SXT images.

The common argument that CMEs exhibit larger angular scales than flares and therefore must be physically independent is indirect and may be misleading. The coronagraph cannot directly observe a CME initiation in the low corona,

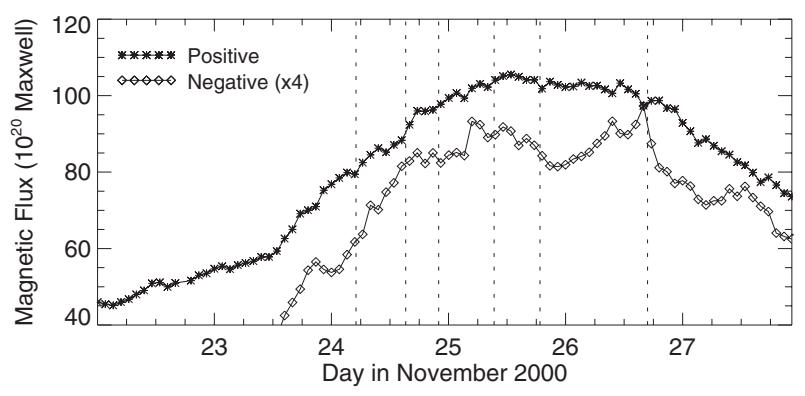

Figure 4. Changes of longitudinal magnetic flux included in the area shown in Figure 3. The dotted lines indicate the times of the six flares $a-f$. 
especially for an event originating away from the limb. Indeed, a CME may start in a compact eruption as shown in one limb event observed by EIT and LASCO [Dere et al., 1997]. We often fail to identify global changes in EUV and $\mathrm{X}$-ray imagery prior to CMEs, as for example in the events discussed here. Concerning the relative timing of the CME onset, we first point out that there are not many studies for impulsive flares with adequate sampling in time. The height-vs-time plots indicating CME initiation prior to the associated flare have been shown mostly for LDEs. In this study, however, we have chosen not to conduct such backprojections because of large extrapolations needed for events away from the limb such as ours; moreover CMEs have 3-d structures and may involve non-radial motions. We simply indicate that some of the CMEs associated with flares $a-f$ can be as fast as the one associated with flare $g$ (closer to the limb) at a given height, since the plane-of-the-sky measurement of LASCO data does not give us the real height. Studies of several other flare-associated CMEs as observed with SXT and TRACE, presented elsewhere [Nitta, 2000], strongly suggest that CME acceleration is most rapid at flare onset. Concerning the heliospheric effects, in addition to the SEP events during November 24-26 as mentioned earlier, the KP index increased to $\geq 6$ on the 26 th and 28 th. CMEdriven shocks are known to be important for these phenomena [Gosling, 1993; Reames, 1999], but we recall that CMEs fast enough to drive shocks tend to be associated with flares. Moreover, Cane [2001] has suggested that flare acceleration makes an important contribution in major proton events.

We emphasize here the possible importance of vigorous magnetic flux emergence, on small spatial scales, in the repeated occurrence of major flares and CMEs, because it implies that the coronal field is always in a high-energy state (cf., Low [1982]). Further analysis of magnetograms may help to understand the recurrent and homologous occurrence of global magnetic restructurings as seen in the flare/CME events reported here.

Acknowledgments. We thank R. Canfield for constructive comments on the manuscript. We acknowledge the useful comments by the anonymous referees, which have tremendously improved the paper. This work has been supported by NASA contract NAS8-00119.

\section{References}

Brueckner, G. E. et al., The Large Angle Spectroscopic Coronagraph (LASCO), Solar Phys., 162, 357-402, 1995

Cane, H. V., Solar flares, fast-drift radio bursts and energetic particles, J. Geophys. Res., submitted, 2001

Delaboudinière, J.-P. et al., EIT: Extreme-Ultraviolet Imaging Telescope for the SOHO mission, Solar Phys., 162, 291-312, 1995
Dere, K. P. et al., EIT and LASCO observations of the initiation of a coronal mass ejection, Solar Phys., 175, 601-612, 1997

Gosling, J. T., The solar flare myth, J. Geophys. Res., 98, 1893718949, 1993

Handy, B. N. et al., The transition region and coronal explorer, Solar Phys., 187, 229-260, 1999

Howard, R. A., D. J. Michels, N. R. Sheeley Jr., and M. J. Koomen, The observation of a coronal transient directed at earth, Astrophys. J., 263, L101-L104, 1982

Kahler, S. W., The morphological and statistical properties of solar X-ray events with long decay times, Astrophys. J., 214, 891-897, 1977

Kahler, S. W., N. R. Sheeley, Jr., and M. Liggett, Coronal mass ejections and associated X-ray flare durations Astrophys. J., 344, 1026-1033, 1989

Kosugi, T. et al., The Hard X-ray Telescope (HXT) for the SolarA mission, Solar Phys., 136, 17-36, 1991

Low, B. C., Self-similar magnetohydrodynamics. I. The $\gamma=4 / 3$ polytrope and the coronal transient, Astrophys. J., 254, 796805, 1982

Low, B. C., Solar activity and the corona, Solar Phys., 167, 217265, 1996

MacQueen, R. M. and R. R. Fisher, The kinematics of solar inner coronal transients, Solar Phys., 89, 89-102, 1983

Munro, R. H., J. T. Gosling, E. Hildner, E. M. MacQueen, A. I. Poland, and C. L. Ross, The association of coronal mass ejection transients with other forms of solar activity, Solar Phys., 61, 201-215, 1979

Nitta, N. V., Observational signatures of the onset of coronal mass ejections, BAAS., 32, 02.76, 2000

Nitta, N. and S. Akiyama, Relation between flare-associated Xray ejections and coronal mass ejections, Astrophys. J., 525, L57-L60, 1999

Reames, D. V., Particle acceleration at the Sun and in the heliosphere, Space Sci. Rev., 90, 413-488, 1999

Scherrer, P. H. et al., The solar oscillations investigation - Michelson Doppler Imager, Solar Phys., 162, 129-188, 1995

Sheeley, N. R., Jr. et al., Coronal changes associated with a disappearing filament, Solar Phys., 45, 377-392, 1975

Sheeley, N. R., Jr., J. H. Walters, Y.-M. Wang, and R. A. Howard, Continuous tracking of coronal outflows: Two kinds of coronal mass ejections, J. Geophys. Res., 104, 24739-24767, 1999

Svestka, Z., Varieties of coronal mass ejections and their relation to flares, Space Sc. Rev., 95, 135-146, 2001

Tsuneta, S. et al., The Soft X-ray Telescope for the Solar-A mission, Solar Phys., 136, 37-67, 1991

H. S. Hudson, Solar Physics Research Corporation, c/o T. Kosugi, Institute of Space and Astronautical Science, 31-1 Yoshinodai, Sagamihara, Kanagawa 229, Japan. (e-mail: hudson@isass1.solar.isas.ac.jp)

N. V. Nitta, Lockheed Martin Solar and Astrophysics Laboratory, Dept/L9-41, B/252, 3251 Hanover Street, Palo Alto, CA 94304. (e-mail: nitta@lmsal.com)

(Received April 3, 2001; revised June 25, 2001; accepted July 18, 2001.) 